\title{
Neuroblastoma in adults: Differential diagnosis of giant retroperitoneal mass
}

\author{
Irving Reyna-Blanco ${ }^{1}$, Iñigo Navarro-Ruesga ${ }^{1 *}$, Rocío Chávez-Pedraya², Ereni Arenas-Manjarrez³ \\ Francisco J. Santa María-Orozco ${ }^{1}$, Gerardo Fernández-Noyola ${ }^{1}$, and Carlos Pacheco-Gahbler ${ }^{1}$ \\ ${ }^{1}$ Department of Urology, Hospital General "Dr. Manuel Gea González;" Mexico City, Mexico; '2Department of Radiology, Hospital HMG Coyoacán, \\ Mexico City, Mexico; ${ }^{3}$ Department of Pathology, Hospital General “Dr. Manuel Gea González," Mexico City, Mexico
}

\begin{abstract}
Neuroblastoma develops from the cells of the primitive neural crest, although being the fourth most frequent cancer in childhood, it is seldom seen in adults, and when found in them, symptoms are usually nonspecific such as abdominal and lumbar pain, therefore, diagnosis is rarely suspected and often delayed. Imaging studies, such as abdominal computed tomography, identify laterality and size of the adrenal mass, which commonly has calcifications; blood work usually does not show abnormalities, excluding functional adrenal tumor. Definitive diagnosis requires histopathological examination. Surgical resection, when feasible, is the treatment of choice. We briefly reviewed diagnostic work-up, histopathological findings, and post-treatment outcome.
\end{abstract}

Key words: Neuroblastoma. Diagnosis. Adrenalectomy. Prognosis.

\section{Introduction}

Neuroblastomas are malignant tumors derived from cells of the primitive neural crest, they represent the fourth most common extracranial malignant tumor in childhood $^{1}$, approximately $90 \%$ of patients present them before 5 years of age, while being extremely rare during adulthood, especially after the third decade of life $^{2}$, with an incidence of 0.12 cases $/$ million $^{3}$, and equal distribution among female and male adults ${ }^{4}$. Reports on adult-onset neuroblastomas can be found in large electronic registries such as the Surveillance, Epidemiology, and End Results (SEER) and the International Neuroblastoma Research Group (INRG) databases. The SEER database, between 1973 and 2002, identified 144 patients $\geq 20$ years old at diagnosis, accounting for $6.1 \%$ of all neuroblastoma patients $(\mathrm{n}=2054)$. The INRG database identified 200 patients (2.3\%) between ages 10 and 21 years, from 1990 to $2002^{4}$.

When found in adults, symptoms are non-specific ${ }^{4}$ often delaying diagnosis, besides, laboratory tests are usually normal, and imaging studies show an undetermined adrenal mass, which may be a functional or non-functional adenoma, pheochromocytoma, adrenal carcinoma, sarcoma, T4 renal tumor, and metastasis, among others, therefore wrongful diagnosis is easy to conjecture. The latter is highly relevant because diagnosis is often delayed and disease stage determines the prognosis.

\section{Correspondence:}

*Iñigo Navarro-Ruesga,

E-mail: inigo.navarro.r@gmail.com

DOI: 10.24875/HGMX.200000811
Date of reception: 07-09-2020

Date of acceptance: 15-11-2020

Available online: $29-07-2021$

Rev Med Hosp Gen Mex. 2021;84(3):136-139

www.hospitalgeneral.mx 0185-1063/@ 2020 Sociedad Médica del Hospital General de Mexico. Published by Permanyer. This is an open access article under the CC BY-NC-ND license (http://creativecommons.org/licenses/by-nc-nd/4.0/). 


\section{Case report}

A 35-year-old female, without medical history, seeks medical consultation at the emergency department with intermittent, 3-month, mild left lumbar pain, with irradiation to ipsilateral thigh. Contrast-enhanced abdominal computed tomography (CT) was performed (Fig. 1), finding a large, lobulated, solid left adrenal mass with heterogeneous enhancement. Blood analysis excluded a functional adrenal tumor.

The patient underwent left open radical adrenalectomy without complications. Pathology report: $14 \times 11 \times$ $10 \mathrm{~cm}$ tumor, $1100 \mathrm{~g}$, poorly differentiated neuroblastoma, positive lymphovascular invasion, with non-disrupted capsule (Figs. 2 and 3) confirmed by immune histochemical assay (Table 1).

Metaiodobenzylguanidine single-photon emission CT/CT was performed at 6-month follow-up (Fig. 4), no local recurrence or metastasis found.

\section{Discussion}

Very rarely, neuroblastomas can be diagnosed in adults $^{2}$. Teenagers and adults with neuroblastoma have a worse outcome than children ${ }^{5}$.

As in the case described above, symptoms are typically non-specific, with back and abdominal pain being the most common 4 . Persistence of these symptoms especially in young adults without relevant medical history, as our patient, should be followed by appropriate imaging studies in an attempt to make an appropriate diagnosis. Laterality of the mass, its size, and the presence of calcifications can be observed by abdominal CT, the latter are seen in $80-90 \%$ of cases $^{6}$, although heterogeneous enhancement was found in our patient, no calcifications were identified. As compared to children, a higher proportion of adults have metastatic disease at diagnosis: $75 \%$, whereas in children, only $40-55 \%$ have Stage 4 disease at diagnosis ${ }^{7}$. This might be related to the delay in diagnosis of adult-onset neuroblastoma. Although our patient presented with a huge tumor, no metastases were found.

Amplification of MYCN oncogene, mutations in anaplastic lymphoma kinase, and segmental chromosomal alterations are genetic alterations that can be found in neuroblastoma cells ${ }^{8}$. Definitive diagnosis requires histopathological examination, the finding of sheets of Homer-Wright pseudorosettes, consisting of neuroblasts surrounding eosinophilic neuropil, is a pathognomonic feature $^{9}$. Differential diagnosis of large retroperitoneal
Table 1. Immunohistochemical assay result confirms neuroblastoma diagnosis. immunohistochemical assay

\begin{tabular}{|l|l|}
\hline Antigen & Result \\
\hline Synaptophysin & Positive \\
\hline Chromogranin & Positive \\
\hline Ki67 & Positive \\
\hline
\end{tabular}

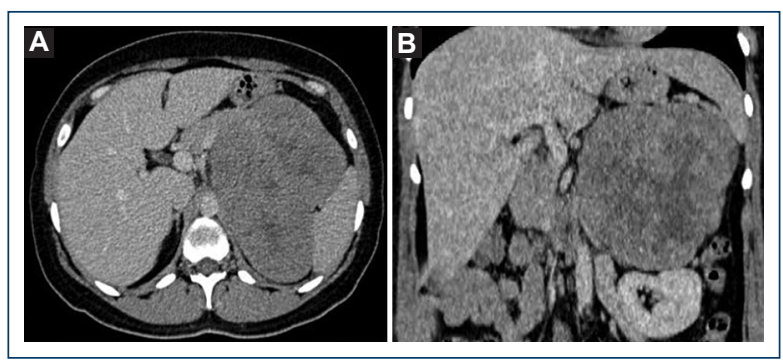

Figure 1. Contrast-enhanced abdominal computed tomography showing. A: axial view of a heterogeneous retroperitoneal large mass (measuring $15 \times 14 \times 12 \mathrm{~cm}$ ). B: giant adrenal mass crossing the midline and displacing adjacent organs and vessels seen in a sagittal view.

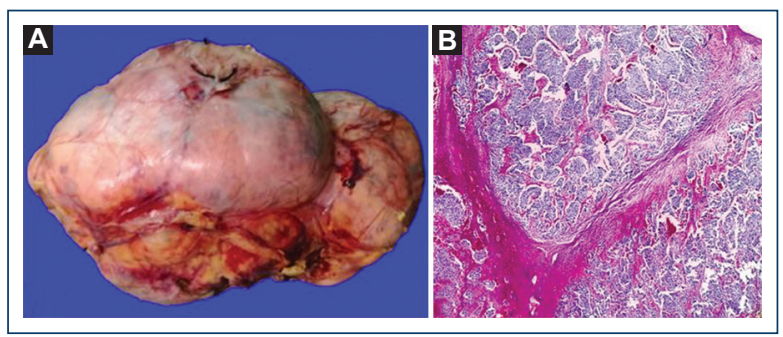

Figure 2. A: surgical specimen. B: hematoxylin and eosin stain $\times 10$, confined malign neoplasm of neuroblastic origin, composed of cell nests surrounded by fibrous septa.

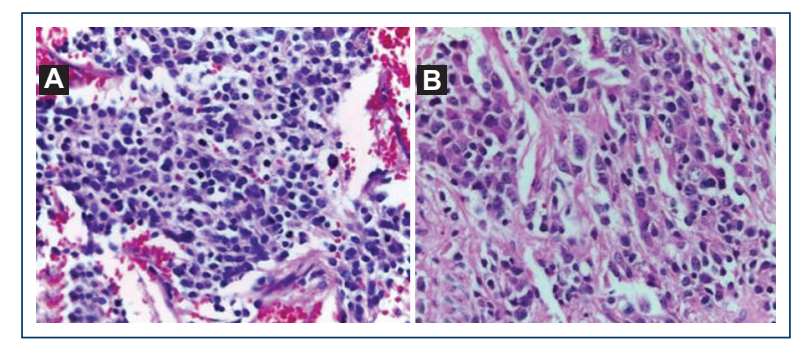

Figure 3. A: hematoxylin and eosin (H-E) stain $\times 40$, cellular characteristics are identified, a large number of neuroblasts are seen at different stages of cellular maturation, ranging from small cells with salt and pepper nucleus to larger cells with an eccentric nucleus and a prominent nucleolus as well as abundant eosinophilic cytoplasm. B: H-E stain $\times 40$, a large number of neuroblasts are seen in different cellular maturation stages in addition to neuropil. 


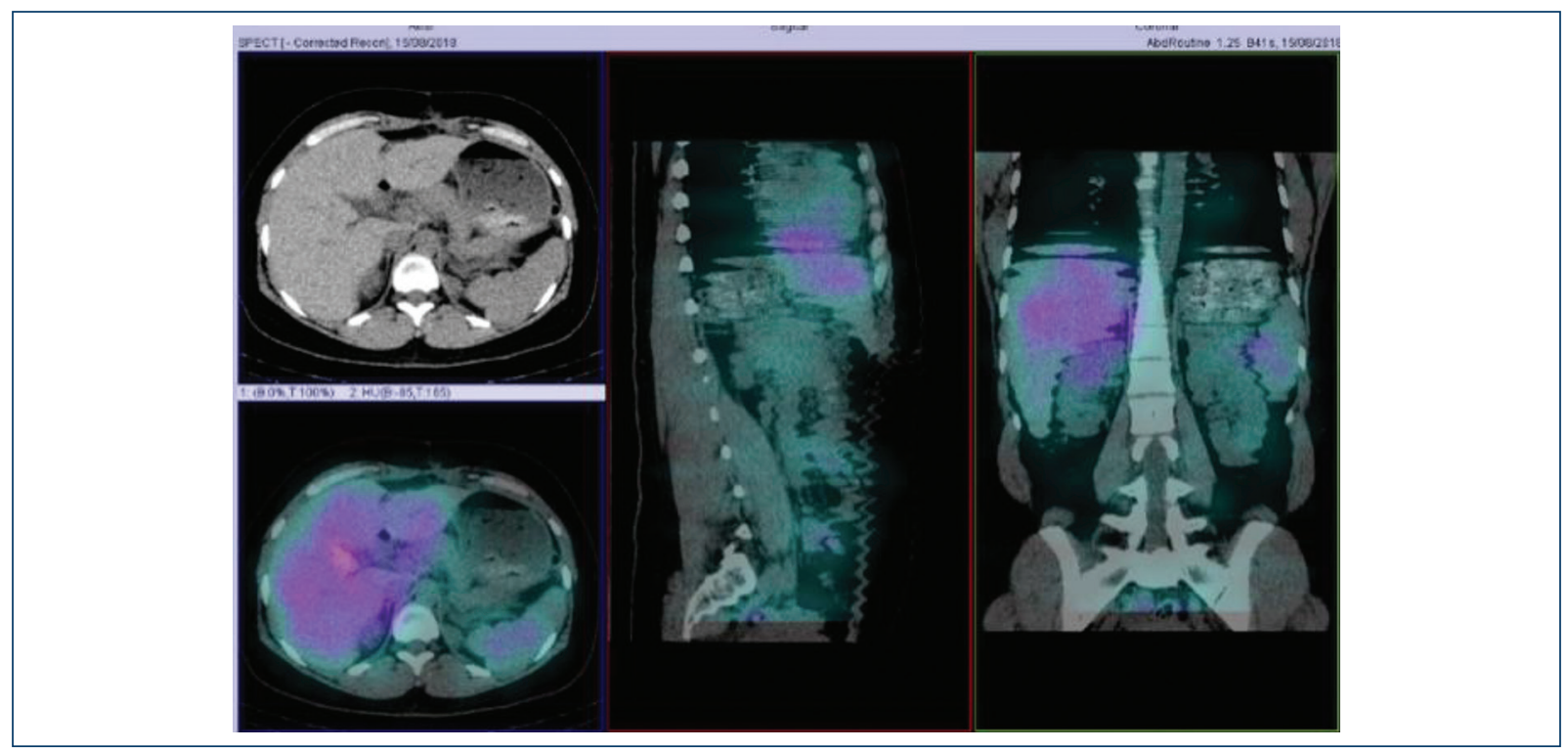

Figure 4. Metaiodobenzylguanidine single-photon emission computed tomography (CT)/CT, no local recurrence or metastasis found.

masses includes retroperitoneal sarcoma, adrenal myelolipoma, retroperitoneal teratoma, and mesenchymal tumors.

A study by the Memorial Sloan Kettering Cancer Center analyzing the largest follow-up cohort of neuroblastoma in adults was carried out between 1979 and 2015, including 44 adult patients, between ages 18 and 71 years. Patients were classified according to the International Neuroblastoma Staging System (INSS), five patients were classified as Stage 1,1 as Stage 2, 5 as Stage 3 , and 33 as Stage 4 . The study revealed that locoregional disease (INSS Stages 1, 2, and 3) had a 10-year progression-free survival (PFS) of $35.4 \%$ and an overall survival (OS) of $61.4 \%$; while patients with metastatic disease (INSS stage 4) at diagnosis had a 5 -year PFS of $9.7 \%$, being statistically significant ${ }^{3}$. For those patients with localized, resectable disease, surgical resection alone is usually curative, and chemotherapy is an effective salvage therapy for those with relapse after resection?. Furthermore, the COG P9641 study demonstrated a 5 -year OS rate after surgical resection alone of $99 \%$ for patients with INSS Stage 1 and $96 \%$ for those with asymptomatic INSS $2 \mathrm{~A}$ or $2 \mathrm{~B}$ tumors $^{10}$.

Taking into account that our patient was classified as INSS Stage 1 (low risk), we should expect a favorable prognosis in the short and medium term. Both, clinical suspicion and carrying out an adequate and complete diagnostic work-up are of utmost importance considering that prognosis is determined by tumor stage and presence of metastases, and when feasible, surgical resection is the treatment of choice.

\section{Acknowledgments}

To the urology and pathology departments of Hospital General Dr. Manuel Gea González.

\section{Funding}

The authors declare that does not exist funding.

\section{Conflicts of interest}

The authors declare that does not exist a conflict of interest.

\section{Ethical disclosures}

Confidentiality of data. The authors declare that they have followed the protocols of their work center on the publication of patient data.

Protection of human and animal subjects. The authors declare that no experiments were performed on humans or animals for this study.

Right to privacy and informed consent. The authors have obtained the written informed consent of the patients or subjects mentioned in the article. The corresponding author is in possession of this document. 


\section{References}

1. Maris JM. Recent advances in neuroblastoma. $N$ Engl $J$ Med. 2010;362:2202-11.

2. Gupta $P$, Maiti A, Aich RK, Deb AR. Adrenal neuroblastoma in an adult J Cancer Res Ther. 2013;9:96-8.

3. Suzuki M, Kushner BH, Kramer K, Basu EM, Roberts SS, Hammond WJ, et al. Treatment and outcome of adult-onset neuroblastoma. Int J Cancer. 2018;143:1249-58

4. Esiashvili N, Goodman M, Ward K, Marcus RB Jr., Johnstone PA. Neuroblastoma in adults: incidence and survival analysis based on SEER data. Pediatr Blood Cancer. 2007;49:41-6.

5. Moroz V, Machin D, Faldum A, Hero B, lehara T, Mosseri V, et al. Changes over three decades in outcome and the prognostic influence of age-at-diagnosis in young patients with neuroblastoma: a report from the international neuroblastoma risk group project. Eur J Cancer. 2011;47:561-71.
6. Lonergan GJ, Schwab CM, Suarez ES, Carlson CL. Neuroblastoma, ganglioneuroblastoma, and ganglioneuroma: radiologic-pathologic correlation. Radiographics. 2002;22:911-34.

7. De Bernardi B, Mosseri V, Rubie H, Castel V, Foot A, Ladenstein R, et al. Treatment of localized resectable neuroblastoma. Results of the LNESG1 study by the SIOP Europe neuroblastoma group. $\mathrm{Br} \mathrm{J}$ Cancer. 2008:99:1027-33.

8. Matthay KK, Maris JM, Schleiermacher G, Nakagawara A, Mackall CL, Diller L, et al. Neuroblastoma. Nat Rev Dis Primers. 2016;2:16078.

9. Weinstein JL, Katzenstein HM, Cohn SL. Advances in the diagnosis and treatment of neuroblastoma. Oncologist. 2003;8:278-92.

10. Strother DR, London WB, Schmidt ML, Brodeur GM, Shimada H, Thorner $\mathrm{P}$, et al. Outcome after surgery alone or with restricted use of chemotherapy for patients with low-risk neuroblastoma: results of children's oncology group study P9641. J Clin Oncol. 2012; $30: 1842-8$ 